\title{
Rotational dynamics of propane in Na-Y zeolite: A molecular dynamics and quasielastic neutron-scattering study
}

\author{
R. Mukhopadhyay, ${ }^{1, *}$ Ahmed Sayeed, ${ }^{2}$ S. Mitra, ${ }^{1}$ A. V. Anil Kumar, ${ }^{2}$ Mala N. Rao, ${ }^{1}$ S. Yashonath, ${ }^{2,3}$ and S. L. Chaplot ${ }^{1}$ \\ ${ }^{1}$ Solid State Physics Division, Bhabha Atomic Research Centre, Trombay, Mumbai 400 085, India \\ ${ }^{2}$ Solid State and Structural Chemistry Unit, Indian Institute of Science, Bangalore-560012, India \\ ${ }^{3}$ Condensed Matter Theory Unit, JNCASR, Jakkur, Bangalore, India
}

\begin{abstract}
We report results from molecular dynamics (MD) simulations and quasielastic neutron-scattering (QENS) measurements on the rotational dynamics of propane in Na-Y zeolite at room temperature with a loading of four molecules per $\alpha$ cage. Rotational part of the intermediate scattering function $F(Q, t)$ obtained from the MD simulation suggests that rotational motion is faster relative to the translational motion. Various rotational models fitted to the MD data suggest that rotation is isotropic. It is found that the hydrogen atoms lie, on the average, on a sphere of radius $1.88 \pm 0.05 \AA$, which is also the average distance of the hydrogen atoms from the center of mass of the propane molecule. Results from QENS measurements are in excellent agreement with those obtained from MD, suggesting that the intermolecular potential employed in the MD simulation provides a realistic description of propane motion within faujasite. The rotational diffusion constant $D_{R}$ is 1.05 $\pm 0.09 \times 10^{12} \mathrm{sec}^{-1}$ from the QENS data, which may be compared with that obtained from the MD data $\left(0.82 \pm 0.05 \times 10^{12} \mathrm{sec}^{-1}\right)$.
\end{abstract}

\section{INTRODUCTION}

Zeolites are well-known molecular sieves and are used extensively in petroleum industries for separation of hydrocarbons [1-3]. For this reason, as well as for their intrinsic theoretical interest, the adsorption and transport of molecules, especially hydrocarbons in zeolites, are widely investigated experimentally and theoretically [4-10]. An understanding of the molecular sieve property of the zeolites requires an understanding of the translational diffusivity $D$ of the polyatomic molecules. A number of factors such as the molecular shape, its interaction with the zeolite, the temperature, etc., determine the magnitude as well as the anisotropic character of self-diffusivity. A number of experimental methods such as quasielastic neutron scattering (QENS), pulsed field gradient NMR are available to measure self-diffusivity [4,5,11-13]. Computational approaches such as molecular dynamics have provided valuable insight into self-diffusivity [14-16]. The results of these three methods of investigation are generally found to be in good agreement for a number of zeolite-sorbate systems [2,17-19]. In recent times, there have been studies that have investigated the role of rotational motion. In certain guest-zeolite systems, it has been observed that there is a strong translational-rotational coupling [20]. It is therefore important to investigate the rotational motion of polyatomics especially hydrocarbon molecules within the cavities of zeolites. Jobic and coworkers [7] have carried out such investigations in recent times.

Recently, we carried out a QENS and molecular dynamics (MD) investigation into the translational motion of propane in zeolite faujasite $[21,22]$. This study indicated that the ro-

\footnotetext{
*Corresponding author.

Email address: mukhop@apsara.barc.ernet.in
}

tational diffusion of propane is significantly faster than the translational diffusion and there is no translation-rotation coupling. This fast diffusion is not expected to be observed with QENS spectrometer at Dhruva with energy resolution of $200 \mu \mathrm{eV}$ [23]. Cohen de Lara and Kahn [24] found that methane performed fast rotation in zeolite $\mathrm{Na}-\mathrm{A}$. Here we report a study of the rotational diffusion of propane in $\mathrm{Na}-\mathrm{Y}$ using the triple-axis spectrometer (TAS) at Dhruva [25] which has a much wider energy window. These results are compared with the analysis of molecular dynamics trajectories.

\section{DETAILS OF MOLECULAR DYNAMICS SIMULATIONS}

Molecular dynamics simulations of propane molecules confined to zeolite Na-Y have been carried out in the microcanonical ensemble. The simulation cell consists of one unit cell of Na-Y zeolite with 32 propane molecules at a loading of four molecules per supercage. Zeolite $\mathrm{Na}-\mathrm{Y}$ or faujasite crystallizes in $F d 3 m$ space group with $a=24.8536 \AA$ [26]. Calculations are carried out on a faujasite with $\mathrm{Si} / \mathrm{Al}$ ratio of $\sim 3.0$ with composition $\mathrm{Na}_{48} \mathrm{Al}_{48} \mathrm{Si}_{144} \mathrm{O}_{384}$. This composition is somewhat higher than that of the actual sample $(\mathrm{Si} / \mathrm{Al}=$ 2.13) used in the experiment. However, this difference is not expected to be significant for the results of the propane dynamics reported here. Cubic periodic conditions are used in all three directions. Zeolite atoms are assumed to be fixed in the simulation. The rotational degrees of freedom are modeled using quaternion formalism [27]. The propane molecules were considered as rigid. Both translational and rotational equations are integrated using the Gear predictorcorrector algorithm. An integration time step of $1 \mathrm{fs}$ is found adequate to get good energy conservation. We have carried out $\mathrm{MD}$ runs at $300 \mathrm{~K}$. A production run of $1 \mathrm{~ns}$ duration has been used to obtain averages after an initial equilibration period of 200 ps. The intermolecular potential parameters 

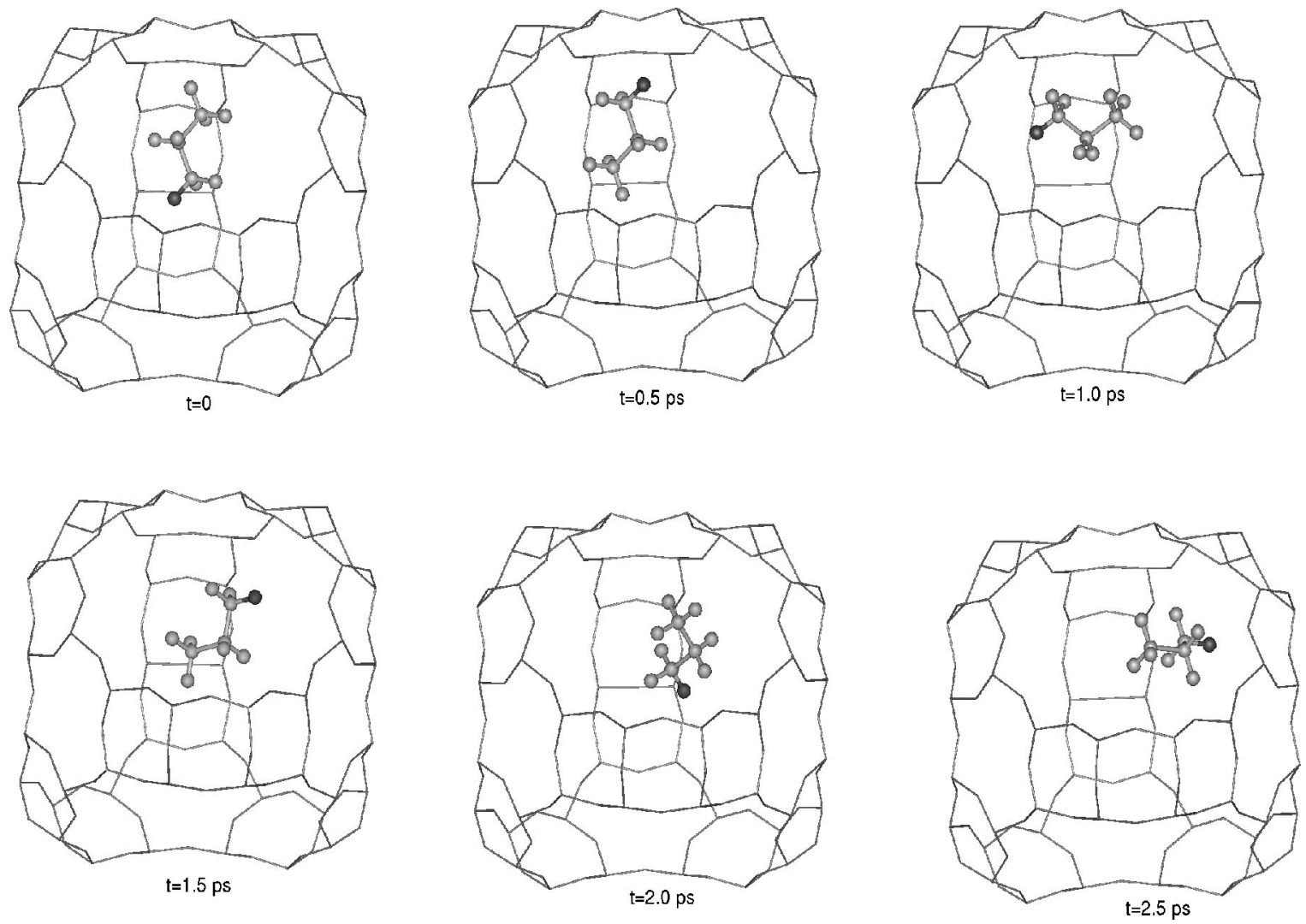

FIG. 1. Snapshots from molecular dynamics to show the translational and rotational motion. A single hydrogen of the $\mathrm{CH}_{3}$ group in propane is shown darker so as to distinguish it from others in order to be able to recognize rotation when it occurs. Rotation through an angle $\pi$ can be seen within 0.5 ps. During this period little or no translation is seen.

between propane and zeolite atoms are taken from the literature $[28,29]$. Lorentz-Berthelot combination rule is used to get the cross or mixed terms.

The potentials are of the 6-12 Lennard-Jones form:

$$
\phi(r)=4 \varepsilon\left[\left(\frac{\sigma}{r}\right)^{12}-\left(\frac{\sigma}{r}\right)^{6}\right]
$$

An all atom model is used for propane, i.e., the hydrogen atom interactions are considered explicitly. The $\mathrm{C}$ and $\mathrm{H}$ atoms of the propane molecules are assumed to interact only with the oxygen atoms of the zeolite framework and with the extra framework cations $(\mathrm{Na})$. The $\mathrm{Si}$ and $\mathrm{Al}$ atoms in the zeolite host are well inside the framework and are largely shielded by the surrounding oxygens leading to negligible short-range interaction of these with the guest molecules. Table I lists the potential parameters for the propane-propane and propane-zeolite interactions.

\section{EXPERIMENTAL DETAILS}

The Na-Y sample used had a Si/Al ratio $\sim 2.13$ as determined by x-ray absorption spectroscopy. Accordingly, we have assumed a unit cell composition of $\mathrm{Na}_{61} \mathrm{Al}_{61} \mathrm{Si}_{131} \mathrm{O}_{384}$. The unit cell parameter varies only slightly for variations in $\mathrm{Si} / \mathrm{Al}$ ratio [26]. The sample $\mathrm{x}$-ray diffraction pattern shows good crystallinity and scanning electron microscope pictures show an average particle size of about $0.5 \mu \mathrm{m}$. The sample was first thoroughly dehydrated by evacuating for a period of about 12 hours to $10^{-4} \mathrm{~Pa}$ at a temperature of $623 \mathrm{~K}$. It was then transferred into two identical slab shaped aluminum containers in ultra-high pressure nitrogen atmosphere. Each sample had dimensions of $6 \mathrm{~cm}$ in diameter and $0.5 \mathrm{~cm}$ in thickness. It was loaded with propane gas (purity $>99.9 \%$ ) to saturation at ambient pressure. The loading was estimated gravimetrically to be $130 \mathrm{mg} / \mathrm{g}$, which in this case turns out to be about 4.1 molecules per zeolitic supercage ( $\alpha$ cage).

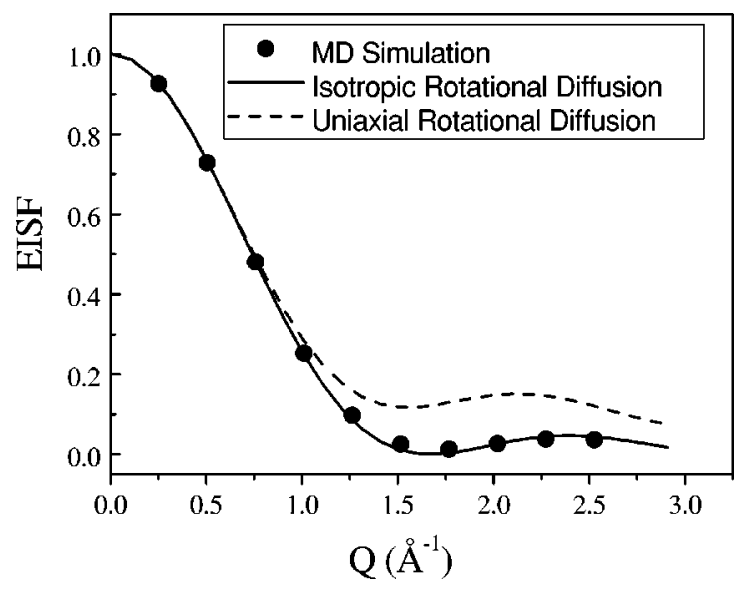

FIG. 2. Variation of EISF as obtained from MD simulation data with Q. Lines are the theoretical EISF for different models (see text). 

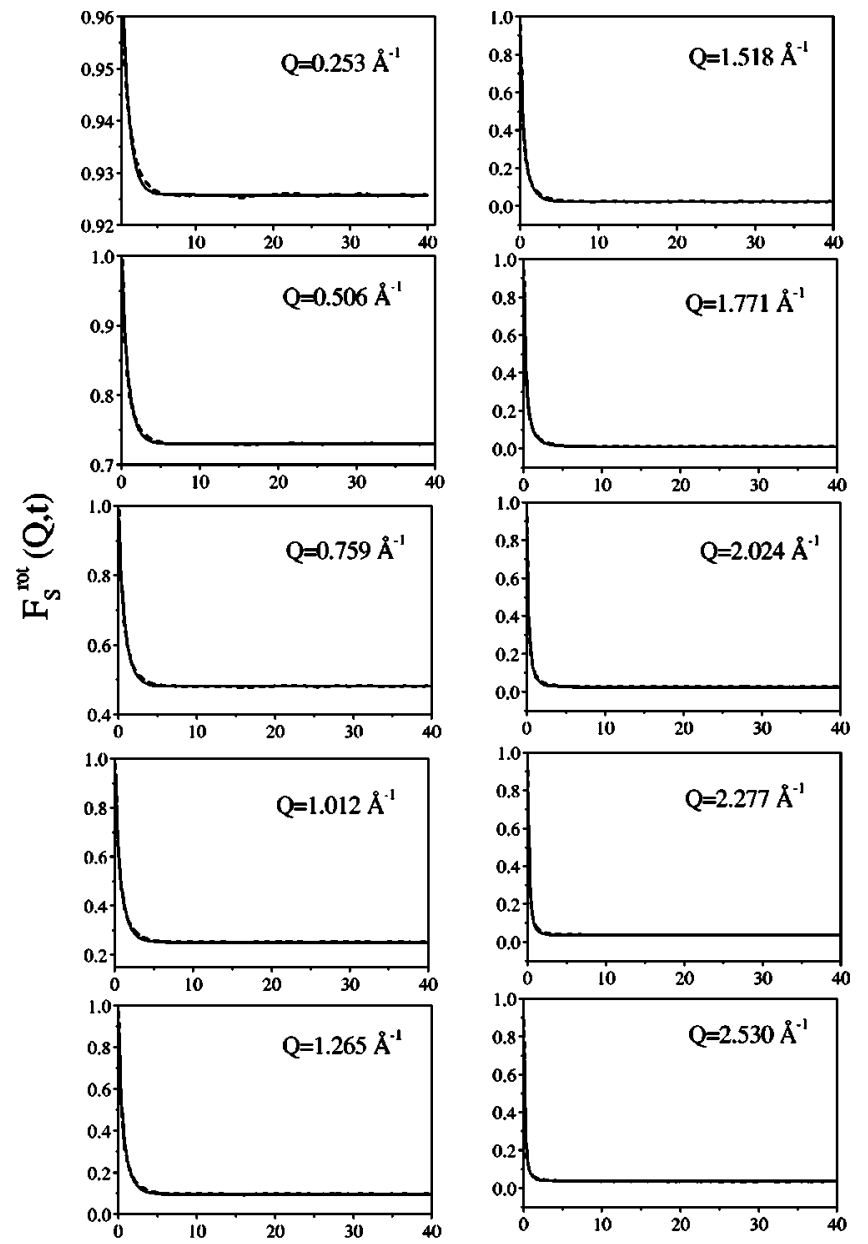

$\mathrm{t}$ (ps)

FIG. 3. $F_{s}^{\text {rot }}(\mathbf{Q}, t)$ vs time at $300 \mathrm{~K}$. Dashed lines are the data from MD simulation and solid lines are the fit with the isotropic rotational diffusion model.

The quantity of propane present in the sample was independently estimated also from neutron transmission measurements and it was found to be very close to that of the loading amount. The QENS experiments were performed using the TAS [25] at the Dhruva reactor at Trombay. TAS was used in the inverted geometry. Incident energy $\left(E_{i}\right)$ was varied and final energy $\left(E_{f}\right)$ was kept fixed. At $E_{f}=20 \mathrm{meV}$, TAS pro-

TABLE I. Potential parameters for propane-propane and propane-Na-Y interactions.

\begin{tabular}{lcc}
\hline \hline Type & $\sigma(\AA)$ & $\epsilon(\mathrm{kJ} / \mathrm{mol})$ \\
\hline $\mathrm{C}-\mathrm{C}$ & 3.448 & 0.3874 \\
$\mathrm{H}-\mathrm{H}$ & 2.980 & 0.0419 \\
$\mathrm{C}-\mathrm{H}$ & 2.920 & 0.2054 \\
$\mathrm{C}-\mathrm{O}$ & 2.996 & 0.7067 \\
$\mathrm{C}-\mathrm{Na}$ & 3.409 & 0.1981 \\
$\mathrm{H}-\mathrm{O}$ & 2.763 & 0.2324 \\
$\mathrm{H}-\mathrm{Na}$ & 3.175 & 0.0405 \\
\hline \hline
\end{tabular}

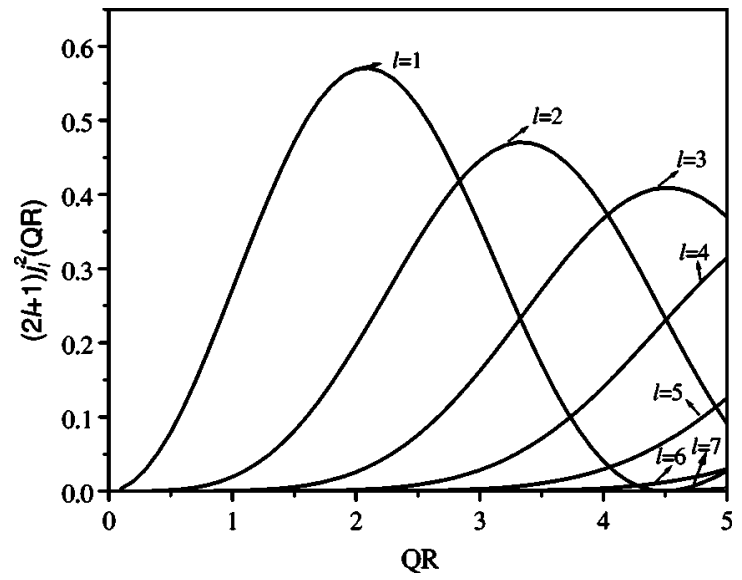

FIG. 4. Variation of quasielastic structure factor, (2l $+1) j_{l}^{2}(\mathbf{Q} R)$, with $\mathbf{Q} R$ for different $l$ (see text).

vides energy resolution [full width at half-maximum (FWHM)] of $\sim 3.3 \mathrm{meV}$ at elastic position in the $Q$ range of $0.8-2.5 \AA^{-1}$, as obtained from measurements on a standard vanadium sample.

The quasielastic spectra were recorded in the wave vector transfer $(Q)$ range of $0.8-2.5 \AA^{-1}$ at $300 \mathrm{~K}$ for both bare zeolite and propane loaded zeolite samples. Spectra from the bare zeolite sample were used to estimate the contribution of bare zeolite to the total spectra.

\section{THEORETICAL ASPECTS}

In a neutron-scattering experiment, the measured intensity is proportional to the double-differential cross section [30]
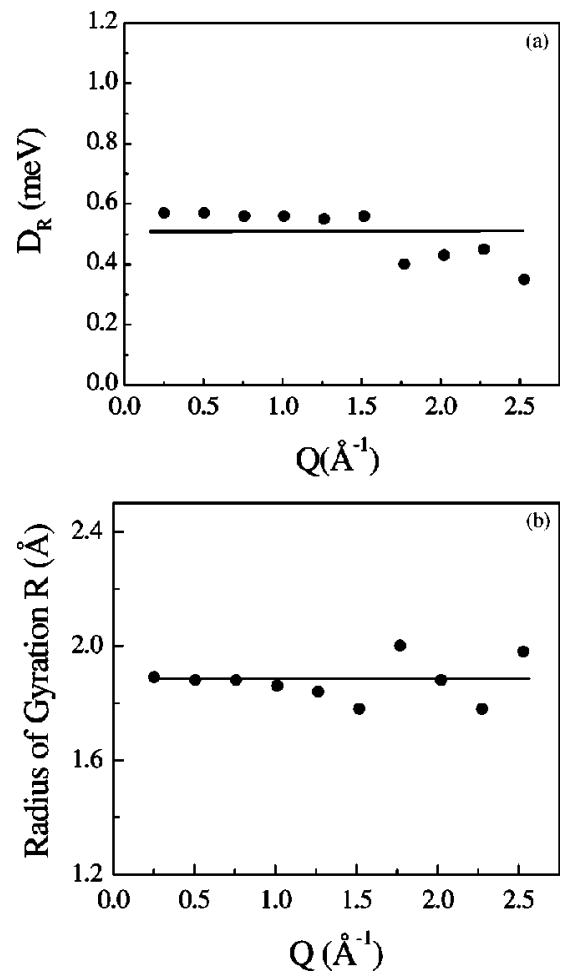

FIG. 5. (a) Diffusion constant $D_{R}$ and (b) radius of gyration $R$ as obtained from fitting the MD simulation data with the isotropic rotational diffusion model at different $\mathbf{Q}$ values. 


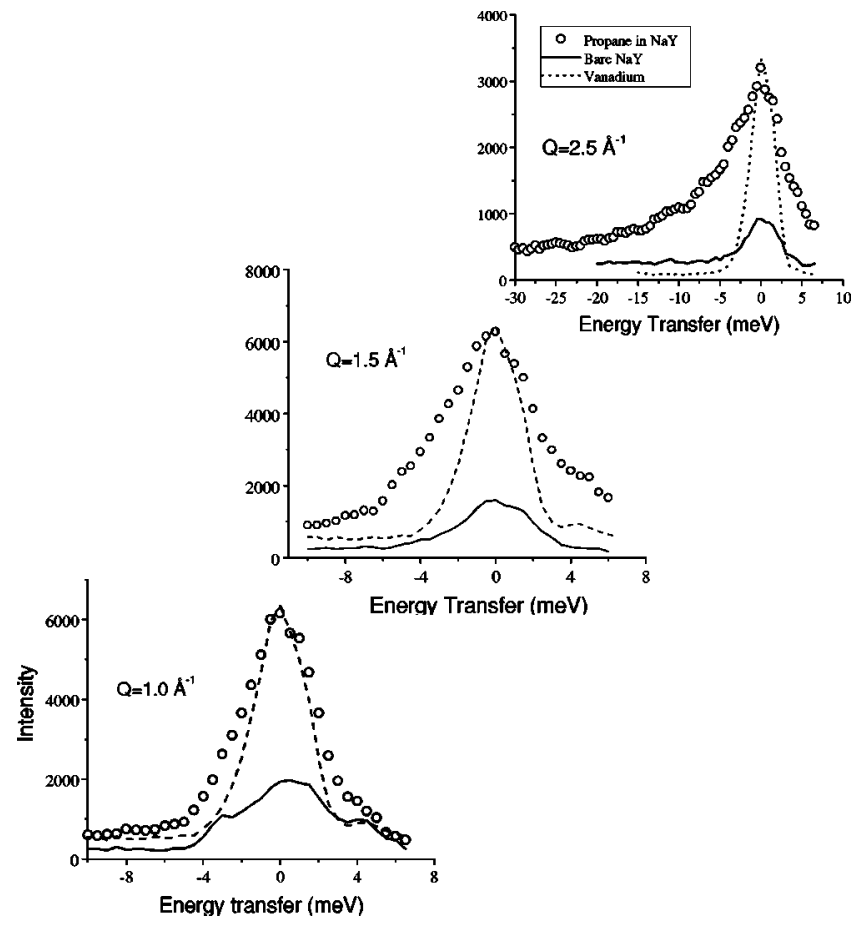

FIG. 6. Measured neutron intensity vs energy transfer at different constant $\mathbf{Q}$, for dehydrated $\mathrm{Na}-\mathrm{Y}$ zeolite, propane-loaded $\mathrm{Na}-\mathrm{Y}$ zeolite and vanadium sample that gives the resolution function of the instrument.
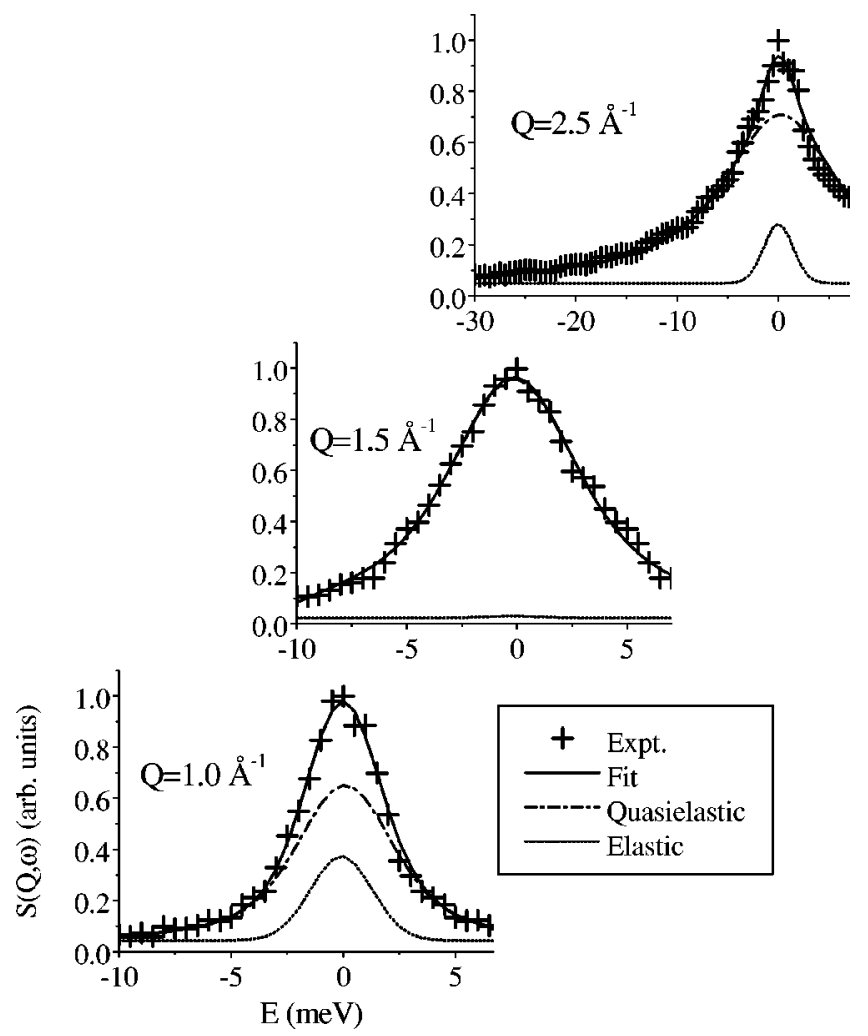

FIG. 7. Experimental $S(\mathbf{Q}, \omega)$ (symbols) and the fit (solid line) using Eq. (10). Separated elastic (dotted) and quasielastic (dashed line) components at different $\mathbf{Q}$ values are also shown.
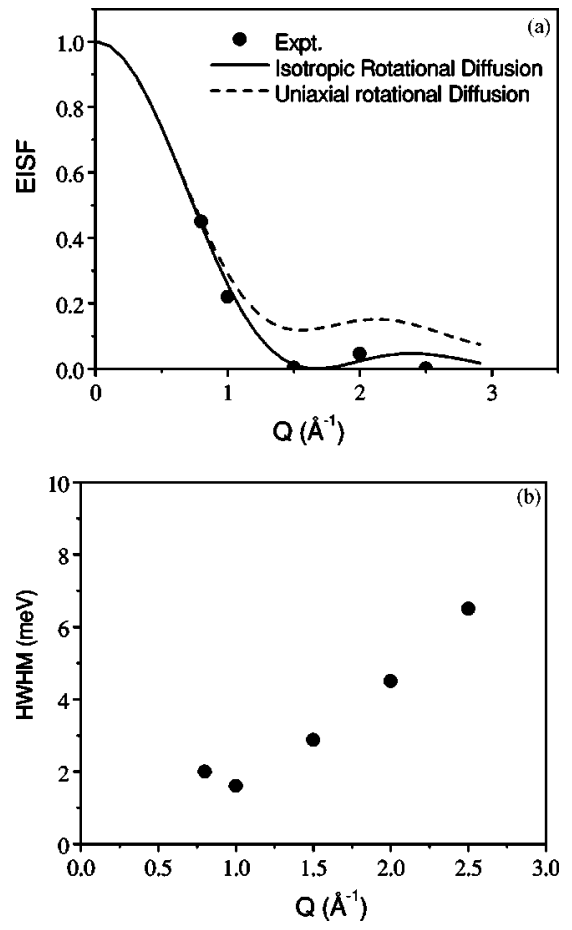

FIG. 8. (a) Variation of EISF as obtained from QENS data (symbols) with $\mathbf{Q}$. The theoretically calculated EISF assuming different models (see text) are shown by lines; (b) the $\mathbf{Q}$ dependence of HWHM of the Lorentzian function $\Gamma(\mathbf{Q})$ obtained from the experimental QENS data.

$$
\frac{\partial^{2} \sigma}{\partial \omega \partial \Omega} \propto \frac{\mathbf{k}}{\mathbf{k}_{0}}\left[\sigma_{\mathrm{coh}} S_{\mathrm{coh}}(\mathbf{Q}, \omega)+\sigma_{\mathrm{inc}} S_{\mathrm{inc}}(\mathbf{Q}, \omega)\right],
$$

where $\sigma$ is the total scattering cross section, $S(\mathbf{Q}, \omega)$ is known as the scattering law and the subscripts coh and inc denote the coherent and incoherent components. $\mathbf{k}$ and $\mathbf{k}_{0}$ are the initial and final wave vectors and $\mathbf{Q}=\mathbf{k}-\mathbf{k}_{0}$ known as wave vector transfer, $\omega$ is the angular frequency corresponding to energy transfer $\hbar \omega=E_{i}-E_{f}$. For a hydrogenous system the measured neutron intensity comes almost entirely from the incoherent scattering of neutrons from hydrogen atoms because of its large scattering cross section $\left[\sigma_{\text {inc }}(H)\right.$ $\sim 80$ barns compared to its coherent part of 1.7 barns, total cross section for a $\mathrm{C}$ atom: 5.5 barns]. Then, Eq. (2) can be written as

$$
\frac{\partial^{2} \sigma}{\partial \omega \partial \Omega} \propto \frac{\mathbf{k}}{\mathbf{k}_{0}}\left[\sigma_{\text {inc }} S_{\text {inc }}(\mathbf{Q}, \omega)\right]
$$

In the case of molecular system, different kinds of motions - translations, rotations, and vibrations - take place simultaneously. It is generally assumed that these three motions are dynamically independent, and can be analyzed separately. This is only an approximation, which is necessary for a mathematically tractable analysis. With this assumption, the total $S(\mathbf{Q}, \omega)$, which is measured in QENS according to Eq. (3), can be expressed as a convolution product in $\omega$ of the different scattering functions [31]: 


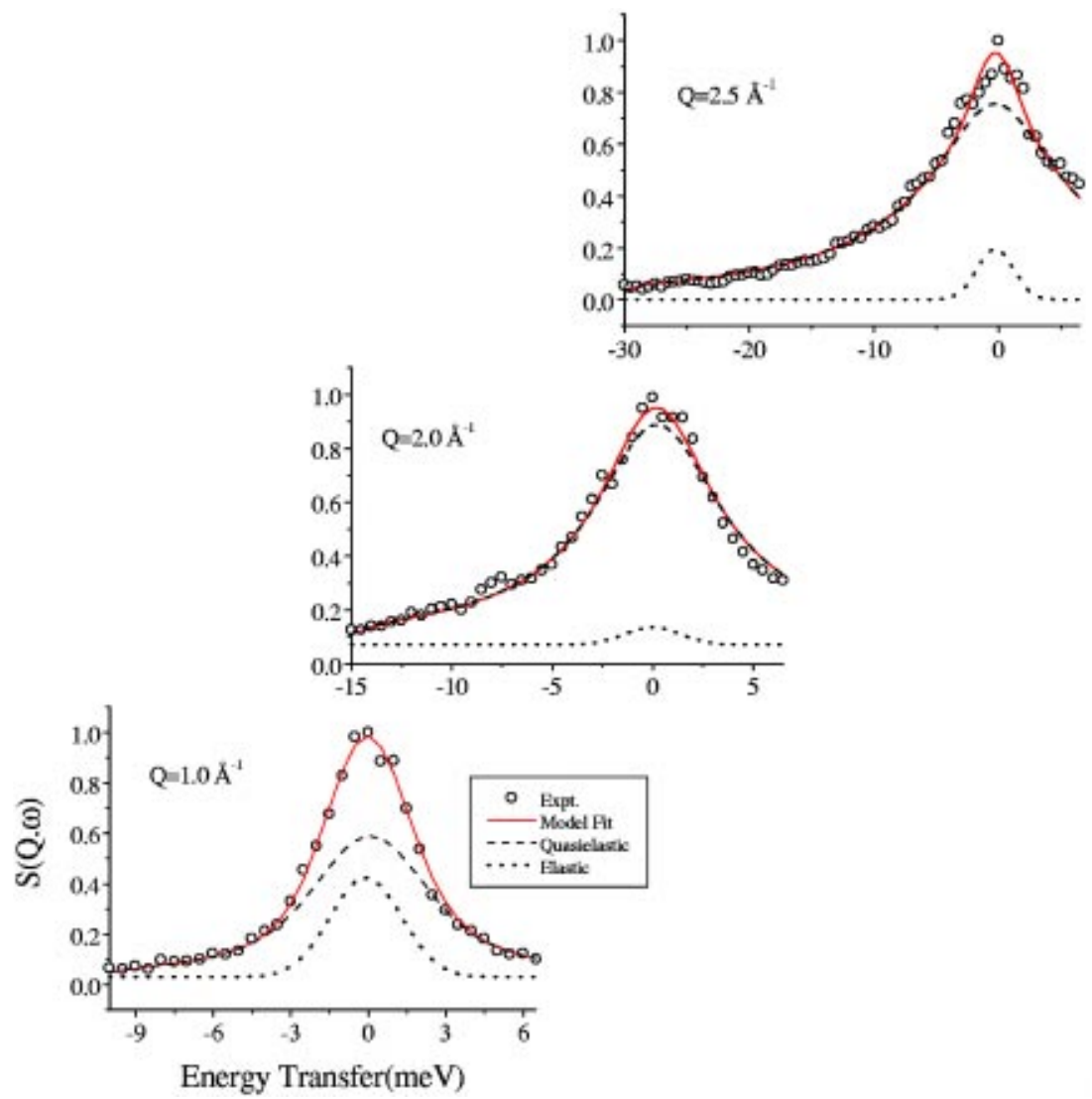

FIG. 9. Fitted $S(\mathbf{Q}, \omega)$ assuming the isotropic rotational diffusion model for propane in $\mathrm{Na}-\mathrm{Y}$ zeolite at different $\mathbf{Q}$ values (see Fig. 7 for information on line styles).

$$
\begin{gathered}
F_{s}^{\mathrm{trans}}(\mathbf{Q}, t)=\langle\exp [i \mathbf{Q} \cdot(\mathbf{R}(t)-\mathbf{R}(0))]\rangle, \\
F_{s}^{\mathrm{rot}}(\mathbf{Q}, t)=\langle\exp [i \mathbf{Q} \cdot(\mathbf{d}(t)-\mathbf{d}(0))]\rangle,
\end{gathered}
$$

as the Debye-Waller factor, where $\left\langle u^{2}\right\rangle$ is the mean-square displacement of the hydrogen atoms. Here we do not consider the inelastic scattering due to the vibrations, which is expected to occur at energies large compared to that considered here in QENS.

The dynamical structure factor $S_{\text {inc }}(\mathbf{Q}, \omega)$ is related to the self part of the intermediate scattering function $F_{s}(\mathbf{Q}, t)$ through a Fourier transform

$$
S_{\mathrm{inc}}(\mathbf{Q}, \omega)=\frac{1}{\pi} \int F_{s}(\mathbf{Q}, t) \exp (-i \omega t) d t .
$$

The intermediate scattering function $F_{s}(\mathbf{Q}, t)$, in the case of incoherent scattering, is a time-correlation function for a single particle and is defined by

$$
F_{s}(\mathbf{Q}, t)=\langle\exp [i \mathbf{Q} \cdot(\mathbf{r}(t)-\mathbf{r}(0)) t]\rangle
$$

where $\mathbf{r}(t)$ and $\mathbf{r}(0)$ are the position vectors of the scatterer (i.e., hydrogen) at time $t$ and $t=0$; the angular brackets indicate ensemble average. Equation (4) can be expressed in terms of intermediate scattering functions for translational and rotational motions as follows (ignoring the vibrational term):

$$
F_{s}(\mathbf{Q}, t)=F_{s}^{\mathrm{trans}}(\mathbf{Q}, t) \cdot F_{s}^{\mathrm{rot}}(\mathbf{Q}, t),
$$

where

where $\mathbf{R}$ is the radius vector of the center of mass of a propane molecule in the fixed frame of reference, and $\mathbf{d}$ is the radius vector of a hydrogen atom with respect to the center of mass of the propane molecule to which it belongs. It may be noted that $\mathbf{r}=\mathbf{R}+\mathbf{d}$.

The rotational scattering law can be written as $[30,31]$

$$
S(\mathbf{Q}, \omega) \propto A(\mathbf{Q}) \delta(\omega)+[1-A(\mathbf{Q})] L(\Gamma, \omega),
$$

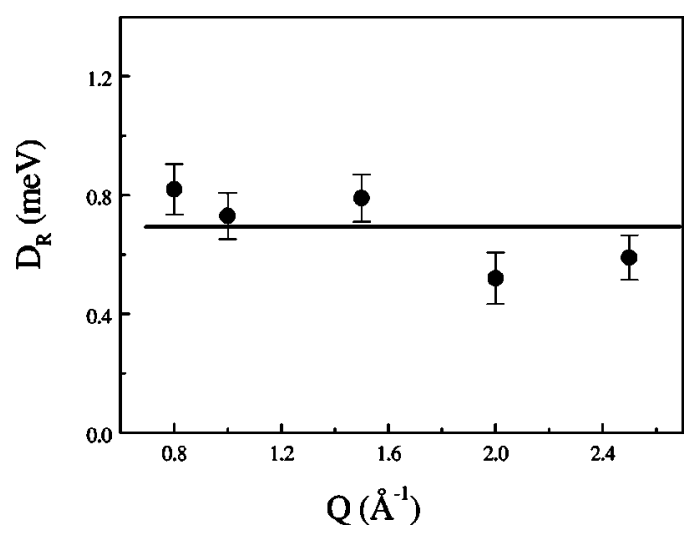

FIG. 10. Variation of diffusion constant $D_{R}$ with $\mathbf{Q}$, as obtained from the fitting shown in Fig. 9. 
where the first term is the elastic part and the second is the quasielastic one. $L(\Gamma, \omega)$ is a Lorentzian function,

$$
L(\Gamma, \omega)=\frac{1}{\pi} \frac{\Gamma}{\Gamma^{2}+\omega^{2}},
$$

where $\Gamma$ is the half width at half-maximum (HWHM) of the Lorentzian function. It is convenient to analyze the data in terms of elastic incoherent structure factor (EISF), which provides information about the geometry of the molecular motions. If $I_{e l}(\mathbf{Q})$ and $I_{q e}(\mathbf{Q})$ are the elastic and quasielastic intensities, respectively, then EISF is defined as [30,31]

$$
\mathrm{EISF}=\frac{I_{e l}(\mathbf{Q})}{I_{e l}(\mathbf{Q})+I_{q e}(\mathbf{Q})} .
$$

Therefore, $A(\mathbf{Q})$ in Eq. (10) is nothing but the EISF.

From the MD trajectories we can readily calculate the intermediate scattering function for the hydrogen atoms with

$$
F_{s}^{\mathrm{rot}}(\mathbf{Q}, t)=\frac{1}{N} \sum_{i=1}^{N}\left\langle\exp \left[i \mathbf{Q} \cdot\left(\mathbf{d}_{i}\left(t+t_{0}\right)-\mathbf{d}_{i}\left(t_{0}\right)\right)\right]\right\rangle,
$$

where $\mathbf{d}_{i}(t)$ is the radius vector of the $i$ th hydrogen at time $t$ and $N$ is the number of hydrogens in a propane molecule, which is equal to 8 . The angular brackets denote (microcanonical) ensemble average; an average over all initial times $t_{0}$. The samples used in the QENS experiment were polycrystalline. Therefore, for the purpose of comparison of QENS and MD results we have to take the powder average of Eq. (13) [32]:

$$
F_{s}^{\mathrm{rot}}(\mathbf{Q}, t)=\frac{1}{\mathbf{N}} \sum_{i=1}^{N}\left\langle\frac{\sin \left(\left|\mathbf{Q} \| d_{i}\left(t+t_{0}\right)-\mathbf{d}_{i}\left(t_{0}\right)\right|\right)}{|\mathbf{Q}|\left|d_{i}\left(t+t_{0}\right)-\mathbf{d}_{i}\left(t_{0}\right)\right|}\right\rangle .
$$

A polyatomic molecule can rotate along any of its axis and there exist different models to describe each of these. Here we discuss a few of these models that are relevant to the present study.

\section{A. Isotropic rotational diffusion}

In this model, molecular reorientation is assumed to take place through random small-angle rotations. Then, on a time average, no most probable orientation exists. For such a case, there is, of course, no difference in the scattering law between a single crystal and powder, since the motion of each individual molecule is already spatially isotropic. It has been shown by Sears [33] that the incoherent scattering law for a scattering particle undergoing isotropic rotational diffusion on the surface of a sphere of radius $a$, with rotational diffusion coefficient $D_{R}$, can be written as

$$
S(\mathbf{Q}, \omega)=A_{0}(\mathbf{Q}) \delta(\omega)+\sum_{l=1}^{l=\infty} A_{l}(\mathbf{Q}) \frac{1}{\pi} \frac{l(l+1) D_{R}}{\left[l(l+1) D_{R}\right]^{2}+\omega^{2}},
$$

with the elastic and quasielastic structure factors, respectively, given by

$$
A_{0}(\mathbf{Q})=j_{0}^{2}(\mathbf{Q} a)
$$

and

$$
A_{l}(\mathbf{Q})=(2 l+1) j_{l}^{2}(\mathbf{Q} a),
$$

where $j_{l}$ is the $l$ th spherical Bessel function.

\section{B. Uniaxial rotational diffusion}

If a cylindrical molecule undergoes rotational diffusion and there is a potential barrier that hinders any rotation about the short axes of the molecule, then the molecule executes uniaxial rotational diffusion and the trajectory of each atom is a circle. The scattering law retains the form of a delta function plus a series of Lorentzian functions [34] but it will now depend on the angle $\theta$ between $\mathbf{Q}$ and the director $\mathbf{n}$ (defining the orientation of the molecule),

$$
\begin{aligned}
S(\mathbf{Q}, \omega)= & J_{0}^{2}(\mathbf{Q} a \sin \theta) \delta(\omega) \\
& +2 \sum_{l=1}^{l=\infty} J_{l}^{2}(\mathbf{Q} \cdot \mathbf{a} \sin \theta) \frac{1}{\pi} \frac{D_{R} l^{2}}{\left(D_{R} l^{2}\right)^{2}+\omega^{2}},
\end{aligned}
$$

where $a$ is the radius of the circle and the $J_{l}$ 's are Bessels functions of the first kind. Here the behavior of the elastic and the quasielastic structure factors strongly depends on angle $\theta$. For a powder sample, one has to take an isotropic average over angle $\theta$. Unfortunately, there exists no formal expression for the average and the result is not simple. However, if the number of equilibrium positions, $N$, is sufficiently large $(\geqslant 6)$, the scattering function for a jump diffusion among $N$ equivalent sites on a circle [35] is nearly identical to that of a uniaxial rotational diffusion [34] and the EISF for uniaxial rotational diffusion model can be written as

$$
A_{0}(\mathbf{Q})=\frac{1}{N} \sum_{n=1}^{N} j_{0}\left(2 \mathbf{Q} \cdot a \sin \left[\frac{n \pi}{N}\right]\right) .
$$

\section{RESULTS AND DISCUSSION}

\section{A. MD simulation}

A snapshot picture of a propane molecule inside the Na-Y supercage and its evolution with time as obtained through MD simulation is shown in Fig. 1. It is evident from these pictures spanning a period of $2.5 \mathrm{ps}$ that the rotational motion is faster than the translational motion. As we directly calculate $F_{s}^{\text {rot }}(\mathbf{Q}, t)$ from the MD data using Eq. (14), it is preferable to work with this function itself, rather than the Fourier transform [Eq. (5)] of it in the frequency domain. $F_{s}^{\text {rot }}(\mathbf{Q}, t)$ is calculated at ten different $\mathbf{Q}$ values, from 0.253 through $2.53 \AA^{-1}$, in multiples of $0.253 \AA^{-1}$. This is the smallest nonzero value of $\mathbf{Q}$ that can be used in the present MD study, because of the requirement that each Cartesian component of $\mathbf{Q}$ must be an integral multiple of $2 \pi / L, L$ being the length of the cubic simulation box. This require- 
ment arises as a consequence of the periodic boundary conditions used in the simulation [36].

If one defines $F_{s}^{\mathrm{rot}}(\mathbf{Q}, \infty)$ as the long time limit of $F_{s}^{\text {rot }}(\mathbf{Q}, \mathbf{t})$, then $F_{s}^{\text {rot }}(\mathbf{Q}, t)$ can be written as an addition of a time dependent term and a limiting $(t=\infty)$ time-independent term as

$$
F_{s}^{\mathrm{rot}}(\mathbf{Q}, t)=F_{s}^{\prime}(\mathbf{Q}, t)+F_{s}^{\mathrm{rot}}(\mathbf{Q}, \infty)
$$

Here, $F_{s}^{\mathrm{rot}}(\mathbf{Q}, \infty)$ is the space Fourier transform of $G_{s}^{\mathrm{rot}}(r, \infty)$, which is the probability that the same particle will be at $\mathbf{r}$ after a time $t=\infty$. Then, $F_{s}^{\text {rot }}(\mathbf{Q}, \infty)$ is the final distribution of the particles in $\mathbf{Q}$ space at long time and has a dimension of a structure factor. Thus, $\mathbf{Q}$ dependence of $F_{s}^{\mathrm{rot}}(\mathbf{Q}, \infty)$ is expected to give the geometry of rotation. Since $F^{\prime}(\mathbf{Q}, t)$ can be approximated by an exponential decay term, $F_{s}^{\text {rot }}(\mathbf{Q}, t)$ can be written as

$$
F_{s}^{\mathrm{rot}}(\mathbf{Q}, t)=B(\mathbf{Q})+C(\mathbf{Q}) e^{-t / \tau} .
$$

One can readily check that this is nothing but the time Fourier transform of Eq. (10) and $B(\mathbf{Q})$ can be identified as EISF as described in Eq. (10). To get the idea of the geometry of rotation or to get the proper model for rotational motion, $F_{s}^{\text {rot }}(\mathbf{Q}, t)$ data from MD are fitted with Eq. (21) at all $\mathbf{Q}$ values keeping $B(\mathbf{Q}), C(\mathbf{Q})$, and $\tau$ as parameters. The variation of $B(\mathbf{Q})$ or EISF at different $\mathbf{Q}$ values obtained from the least squares fit is given in Fig. 2. The theoretically calculated EISF as expected from different models are also shown in Fig. 2. The best description of the data with isotropic rotational diffusion is very apparent. While calculating the theoretical EISF, it is seen that $R=1.88 \AA$ gives the best fit to the data. Once we know the model, we have fitted the $F_{s}^{\mathrm{rot}}(\mathbf{Q}, t)$ data using the isotropic rotational diffusion model directly. The expression for $F_{s}^{\mathrm{rot}}(\mathbf{Q}, t)$ which is the time Fourier transform of Eq. (15), can be written as

$$
F_{s}^{\mathrm{rot}}(\mathbf{Q}, t)=\sum_{l=0}^{\infty}(2 l+1) j_{l}^{2}(\mathbf{Q} R) e^{-l(l+1) D_{R} t}
$$

It is found that the isotropic diffusion model describes the MD data consistently for all the $\mathbf{Q}$ values. The fitted MD simulation data are shown in Fig. 3 where dashed lines are the MD simulation data and solid lines are the fits using Eq. (22). While fitting, up to $l=7$ terms are retained in the expansion of Eq. (22). It is seen with $\mathbf{Q}_{\max } R$ equal to 5, quasielastic structure factor $(2 l+1) j_{l}^{2}(\mathbf{Q} R)$ has a significant contribution only up to $l=7$ as seen from Fig. 4, where $(2 l$ $+1) j_{l}^{2}(\mathbf{Q} R)$ is plotted against $\mathbf{Q} R$ for different values of $l$. The value of $D_{R}$ and the radius of gyration $R$ obtained from the fit are shown in Fig. 5. The obtained value of $D_{R}$ $\left(=0.82 \pm 0.05 \times 10^{12} \mathrm{sec}^{-1}\right)$ agrees quite well with that obtained from the angular velocity autocorrelation function $\left(0.89 \times 10^{12} \mathrm{sec}^{-1}\right)$. The value of the radius of gyration ( $1.88 \pm 0.02 \AA)$ also agrees with the value of the average hydrogen distance from the center of mass of the propane molecule and also with the value of $R$ obtained from fitting the EISF with the model.

\section{B. QENS experiments}

Experiments were carried out in constant $\mathbf{Q}$ mode. Final neutron energy was kept fixed at $20 \mathrm{meV}$ and incident energy was varied. Measured intensity vs energy transfer for the dehydrated $\mathrm{Na}-\mathrm{Y}$ zeolite, propane-loaded Na-Y zeolite, and the resolution function at different $\mathbf{Q}$ values are shown in Fig. 6. Dehydrated zeolite sample did not show any quasielastic broadening, whereas propane-loaded sample did show significant broadening at room temperature indicating the presence of dynamical motion of propane molecules. Raw data were corrected for all geometric factors, second-order reflection from the monochromator, etc. [25] and converted to $S(\mathbf{Q}, \omega)$. To analyze the data, in the first instant the elastic and quasielastic components in the total spectrum were separated, which involves convolution of the model scattering function $S(\mathbf{Q}, \omega)$ [Eq. (10)] with the instrumental resolution function. Then the model parameters were estimated by least squares fit with the experimental data. The fitting parameters were $A(\mathbf{Q})$ (the EISF) and the $\Gamma[(\mathrm{HWHM})$ of the Lorentzian function]. The contribution from the bare zeolite, towards elastic scattering only, was subtracted from the data with the propane-loaded sample. The separated elastic and QE components at different $\mathbf{Q}$ values are shown in Fig. 7 and the extracted EISF as defined in Eq. (12) is shown in Fig. 8(a). The theoretically calculated EISFs for different models (described in Sec. IV above) are shown by lines (solid and dashed) in Fig. 8(a). It is very evident from the figure that the isotropic rotational diffusion model describes the experimental EISF quite well. The variation of the width at different $\mathbf{Q}$ values is shown in Fig. 8(b). This is quite close to that expected for isotropic rotational diffusion. Thereafter, we have used the model $S(\mathbf{Q}, \omega)$ for isotropic rotational diffusion [Eq. $(15)]$ to fit with the experimental QENS data and determine the rotational diffusion constant $D_{R}$. Very good fit was obtained at all the $\mathbf{Q}$ values with the radius of gyration equal to $1.89 \pm 0.02 \AA$, which is also the average distance of the hydrogen atoms from the center of mass of the propane molecule. Fitted spectra at different $\mathbf{Q}$ values are shown in Fig. 9. The variation of $D_{R}$ with $\mathbf{Q}$ is shown in Fig. 10. $D_{R}$ $=1.05( \pm 0.09) \times 10^{12} \mathrm{sec}^{-1}$, the value obtained from QENS experiment (see Fig. 10) is found to be close to the value of $0.82( \pm 0.05) \times 10^{12} \mathrm{sec}^{-1}$ obtained from MD simulation.

\section{CONCLUSIONS}

We have carried out MD simulations and QENS measurements on the rotational motion of propane in $\mathrm{Na}-\mathrm{Y}$ zeolite, with a loading of four molecules per cage, at $300 \mathrm{~K}$. MD simulation showed the presence of very fast rotational diffusion of the propane molecules in Na-Y zeolite. This fast rotational diffusion has been observed through the quasielastic neutron-scattering measurements. The results from MD simulation and QENS measurements agree very closely. Isotropic rotational diffusion model is found to describe both QENS and MD simulation data consistently. Calculation of the rotational diffusion constant $D_{R}$ is carried out from the simulated angular velocity autocorrelation function, as well 
as from the model fitted to the intermediate scattering function. The rotational diffusion constant and the radius of gyration obtained from the QENS studies agree with those obtained from MD. The present work provides for a detailed investigation into the rotational dynamics of propane in zeolite Y.

\section{ACKNOWLEDGMENTS}

A.S., A.V.A., and S.Y. wish to thank IUC-DAEF for support in carrying out this work; Professor M.S. Hegde, IISc, for providing laboratory facilities for sample preparation; and DST, New Delhi, for computational facilities.
[1] R.M. Barrer, Zeolites and Clay Minerals (Academic Press, London, 1978).

[2] J. Kärger and D.M. Ruthven, Diffusion in Zeolites and Other Microporous Solids (Wiley Interscience, New York, 1992).

[3] S.P. Bates and R.A. van Santen, Adv. Catal. 42, 1 (1997).

[4] H. Jobic, M. Bée, and G. J. Kearly, Zeolites 9, 312 (1989).

[5] H. Jobic, M. Bée, J. Caro, M. Bulow, and J. Kärger, J. Chem. Soc., Faraday Trans. 1 85, 4201 (1989).

[6] R. Stockmeyer, Zeolites 4, 81 (1984).

[7] J. Jobic, Phys. Chem. Chem. Phys. 1, 525 (1999).

[8] S. Mitra, R. Mukhopadhyay, A.K. Tripathy, and N.M. Gupta, Appl. Phys. A (to be published).

[9] A.K. Tripathy, A. Sahasrabudhe, S. Mitra, R. Mukhopadhyay, N.M. Gupta, and V.B. Kartha, Phys. Chem. Chem. Phys., 3, 4449 (2001).

[10] R. Tsekov and P.G. Smirniotisov, J. Phys. Chem. B 102, 9385 (1998).

[11] S. Mitra, R. Mukhopadhyay, I. Tsukushi, and S. Ikeda, J. Phys.: Condens. Matter 13, 8455 (2001).

[12] A. Sahasrabudhe, S. Mitra, A.K. Tripathy, R. Mukhopadhyay, and N. M. Gupta, J. Phys. Chem. B (to be published).

[13] L.N. Gergidis, D.N. Theodorou, and H. Jobic, J. Phys. Chem. B 104, 5541 (2000).

[14] A. Schuring, S.M. Auerbach, S. Fritzsche, and R. Haberlandt, J. Chem. Phys. 116, 10890 (2002).

[15] K. Makrodimitris, G.K. Papadopoulos, and D.N. Theodorou, J. Phys. Chem. 105, 777 (2001).

[16] C.R. Kamala, G. Ayappa, and S. Yashonath, Phys. Rev. E 65, 061202 (2002).

[17] H. Jobic, M. Bée, and G.J. Kearly, J. Phys. Chem. 98, 4660 (1994).

[18] H. Jobic, M. Bée, B. Frick, and A. Methivier (unpublished).
[19] R. Q. Snurr and J. Kärger, J. Phys. Chem. B 101, 6469 (1997).

[20] R. Chitra, A.V. Anil Kumar, and S. Yashonath, J. Chem. Phys. 114, 11 (2001).

[21] S. Mitra, R. Mukhopadhyay, A. Sayeed, S. Yashonath, and S. L. Chaplot, Appl. Phys. A (to be published).

[22] A.V. Anil Kumar, A. Sayeed, S. Mitra, R. Mukhopadhyay, S. Yashonath, and S.L. Chaplot J. Phys. Chem. B (to be published).

[23] R. Mukhopadhyay, S. Mitra, S.K. Paranjpe, and B.A. Dasannacharya, Nucl. Instrum. Methods Phys. Res. A 474, 55 (2001).

[24] E. Cohen de Lara and R. Kahn, J. Phys. (Paris) 42, 1029 (1981).

[25] S.L. Chaplot, R. Mukhopadhyay, P.R. Vijayaraghavan, A.S. Deshpande, and K.R. Rao, Pramana-J. Phys. 33, 595 (1989).

[26] A.N. Fitch, H. Jobic, and A. Renouprez, J. Phys. Chem. 90, 311 (1986).

[27] D.J. Evans, Mol. Phys. 34, 317 (1977).

[28] G. Filippini and A. Gavazzotti, Acta Crystallogr., Sect. B: Struct. Sci. 49, 868 (1993).

[29] P. Santikary and S. Yashonath, J. Chem. Soc., Faraday Trans. 88, 1063 (1992).

[30] M. Bée, Quasielastic Neutron Scattering (Adam Hilger, Bristol, 1988).

[31] W. Press, Single Particle Rotations in Molecular Crystals (Springer, Berlin, 1981).

[32] K.W. Herwig, Z. Wu, P. Dai, H. Taub, and F. Y. Hansen, J. Chem. Phys. 107, 5186 (1997).

[33] V.F. Sears, Can. J. Phys. 44, 1999 (1966).

[34] A.J. Dianoux, F. Volino, and H. Hervet, Mol. Phys. 30, 1181 (1975).

[35] J.D. Barnes, J. Chem. Phys. 58, 5193 (1973).

[36] F.R. Trouw, Spectrochim. Acta, Part A 48, 455 (1992). 